\title{
Pregabalin in the Management of Aneurysmal Subarachnoid Hemorrhage
}

\author{
Sotirios Apostolakis ${ }^{1}$ \\ ${ }^{1}$ Department of Neurosurgery, KAT General Hospital of Attica, \\ Kifisia, Greece
}

J Neurosci Rural Pract 2019;10:444-445

Excruciating headache is the most frequent complaint of patients suffering from aneurysmal subarachnoid hemorrhage (ASAH), ${ }^{1}$ which may persist even for about a year following ictus, ${ }^{2}$ requiring long-term medication therapy. ${ }^{1}$

Pain control is of paramount importance in the management of the ASAH patient not only for the achievement of the direct goal of patient comfort but also for the proper management of arterial blood pressure, a key risk factor for aneurysmal rupture. In fact, it is believed that proper analgesia suffices for the satisfactory management of blood pressure in these patients. ${ }^{3}$

Several different approaches have been tested and adopted to address SAH-induced headache, among which the most frequently applied in everyday practice is the administration of primarily a combination of paracetamol/acetaminophen, nonsteroidal anti-inflammatory drugs, and opioid analgesics. ${ }^{4,5}$ Nevertheless, due to the potential side effects of the latter that includes but is not limited to central nervous system (CNS) depression that can confound the neurologic examination, titrating the dosage to meet the individual needs of each patient, while avoiding overdose may be fairly challenging. Regardless of the importance of adequate pain management in ASAH, only a few studies have investigated the potential efficiency of novel compounds or the introduction of adjuvant medication in the already widely applied regimes.

As the aforementioned approach has variable efficiency and the long-term administration of analgesics may cause deleterious side effects, alternatives have been tested, including scalp block in medication-refractory cases. ${ }^{6}$ The latter, while considered highly effective and relatively safe by the authors, nevertheless is an invasive procedure, bearing as such all the potential drawbacks such as pain, local inflammation, and failure of the procedure.

This hard-to-manage headache has been proposed to be secondary to cerebral vasospasm ${ }^{2}$; however, this hypothesis has been challenged by some authors. ${ }^{5}$ Cerebral vasospasm is a frequent complication of ASAH that leads to reduced cerebral blood flow $(\mathrm{CBF})$ as well as to increased
Address for correspondence Sotirios Apostolakis, MD, MRes, 2 Nikis Street, Kifisia 145 61, Greece (e-mail: sotapostolakis@gmail.com).

intracranial pressure (ICP), ultimately resulting in reduced cerebral perfusion pressure and diffuse ischemia.

These alterations in CNS homeostasis may be further aggravated during induction and maintenance of anesthesia, with the group of volatile anesthetics causing an increase in ICP whereas CBF decreases. ${ }^{7}$ On the other hand, the administration of intravenous anesthetics, such as propofol, is related to a dose-dependent decrease in $\mathrm{CBF}$, cerebral metabolic rate of oxygen, and ICP. Hence, meticulous selection of the anesthetic agent and its optimal dosage is required, to minimize the risk for cerebral ischemia.

The aforementioned key topics are addressed by Lionel et $\mathrm{al}^{8}$ in the article entitled "Effect of pregabalin on perioperative headache in patients with aneurysmal subarachnoid hemorrhage: a randomized double-blind placebo-controlled trial." In this study, the authors assessed the analgesic properties of pregabalin on a series of ASAH patients managed surgically. Besides, a statistically significant reduction in pre- and postoperative pain, the authors also found significantly reduced needs for volatile anesthetics intraoperatively in the pregabalin group.

The effectiveness of pregabalin has been poorly investigated in conditions other than neuropathic pain, let alone subarachnoid hemorrhage, while at the same time, there is a growing body of literature providing evidence for its potential adjuvant role in anesthesia induction and maintenance. With these in mind, future research could address, among other, the potential neuroprotective role of pregabalin in ASAH by extending the available investigations on cerebral ischemia, its ability to prevent postoperative neuroinflammation and cognitive decline, as animal studies have suggested, ${ }^{9}$ and most importantly, its potential indirect effect on the surgical outcome of patients with ASAH, through the reduction in the need for anesthetics.

We are all eagerly waiting for more studies that will provide a profound investigation and establish a potent clinical benefit of pregabalin in ASAH, a condition notorious for its high morbidity and mortality rates, even when managed aggressively. 


\section{Funding}

None.

\section{Conflict of Interest}

None declared.

\section{References}

1 Suarez JI, Tarr RW, Selman WR. Aneurysmal subarachnoid hemorrhage. N Engl J Med 2006;354(4):387-396

2 Hong CK, Joo JY, Kim YB, et al. The course of headache in patients with moderate-to-severe headache due to aneurysmal subarachnoid hemorrhage: a retrospective cross-sectional study. Headache 2015;55(7):992-999

3 Suarez JI. Diagnosis and management of subarachnoid hemorrhage. Continuum (Minneap Minn) 2015;21(5 Neurocritical Care):1263-1287
4 Zeiler FA, AlSubaie F, Zeiler K, Bernard F, Skrobik Y. Analgesia in neurocritical care: an international survey and practice audit. Crit Care Med 2016;44(5):973-980

5 Glisic EK, Gardiner L, Josti L, et al. Inadequacy of headache management after subarachnoid hemorrhage. Am J Crit Care 2016;25(2):136-143

6 Venkatesulu KB, Nandhakumar A, Cherian M, et al. Scalp block for management of subarachnoid hemorrhage (SAH)-induced headache. J Neurosurg Anesthesiol 2019;31(3):356-357

7 Priebe HJ. Aneurysmal subarachnoid haemorrhage and the anaesthetist. Br J Anaesth 2007;99(1):102-118

8 Lionel KR, Sethuraman M, Abraham M, Vimala S, Prathapadas $\mathrm{U}$, Hrishi AP. Effect of pregabalin on perioperative headache in patients with aneurysmal sub-arachnoid hemorrhage: a randomized double-blind placebo-controlled trial. J Neurosci Rural Pract 2019;10(3):438-443

9 Kawano T, Eguchi S, Iwata H, et al. Pregabalin can prevent, but not treat, cognitive dysfunction following abdominal surgery in aged rats. Life Sci 2016;148:211-219 